KYUNGPOOK Math. J. 51(2011), 293-300

http://dx.doi.org/10.5666/KMJ.2011.51.3.293

\title{
Path-Connectivity of Two-Interval MSF Wavelets
}

Divya Singh

Department of Mathematics, University of Allahabad, Allahabad - 211 002, India

e-mail : divya_allduniv@yahoo.com

ABSTRACT. In this paper, we obtain that the space $\mathcal{W}_{2}$ of minimally supported frequency wavelets, the supports of whose Fourier transforms consist of two intervals, is path-connected.

\section{Introduction}

The study of topological notion of path-connectedness for the space of orthonormal wavelets was initiated by Dai and Larson [1]. In [9], a similar study has been made and it is obtained that the space $\mathcal{W}^{M}$ of MRA wavelets is path-connected. Latter, in [8], it has been proved that the space $\mathcal{W}^{F}$ of MSF wavelets (s-elementary wavelets) is path-connected. Further, path-connectedness of such spaces of wavelets have been considered for higher dimensions as well $[5,6,8]$.

In this paper, we consider the set $\mathcal{W}_{2}$ of one-dimensional MSF wavelets, the supports of whose Fourier transforms consist of two intervals. These are known to be MRA wavelets [3]. Thus $\mathcal{W}_{2}$ being a subset of $\mathcal{W}^{M}$ derives induced topology on it. It has been observed that the path joining the Shannon wavelet $\psi_{0}$ and a member $\psi$ of $\mathcal{W}_{2}$ not equal to $\psi_{0}$, described in [9], does not lie in $\mathcal{W}_{2}$. Thus, a question of path-connectivity of $\mathcal{W}_{2}$ arises. We answer this question in affirmative.

\section{Pre-Requisites}

Let $L^{1}(\mathbb{R})$ be the collection of all Lebesgue integrable functions on $\mathbb{R}$ and $L^{2}(\mathbb{R})$ be that of all Lebesgue square integrable functions on $\mathbb{R}$. With the usual addition and scalar multiplication of functions together with the inner-product $\langle f, g\rangle$ of $f, g \in$ $L^{2}(\mathbb{R})$ defined by

$$
\langle f, g\rangle=\int_{\mathbb{R}} f(x) \overline{g(x)} d x
$$

Received July 17, 2010; accepted March 7, 2011.

2010 Mathematics Subject Classification: 42C40.

Key words and phrases: Wavelet set, MSF wavelet, Multiresolution analysis. 
$L^{2}(\mathbb{R})$ becomes a Hilbert space. The Fourier transform $\mathcal{F}$ is defined by

$$
\hat{f}(s) \equiv(\mathcal{F} f)(s)=\int_{\mathbb{R}} f(t) e^{-i s t} d t,
$$

where $f \in L^{1}(\mathbb{R}) \cap L^{2}(\mathbb{R})$. This Fourier transform can be extended uniquely to an operator on $L^{2}(\mathbb{R})$.

An orthonormal wavelet (or, a wavelet) in $L^{2}(\mathbb{R})$ is a function $\psi \in L^{2}(\mathbb{R})$ with unit norm such that the family $\left\{2^{n / 2} \psi\left(2^{n} \cdot-l\right): n, l \in \mathbb{Z}\right\}$ constitutes an orthonormal basis for $L^{2}(\mathbb{R})$.

One of the methods of constructing orthonormal wavelets is based on the existence of a family of closed subspaces of $L^{2}(\mathbb{R})$ satisfying certain properties. Such a family is called a multiresolution analysis or, simply an MRA.

Definition 2.1([7]). A sequence of closed subspaces $\left(V_{j}\right)_{j \in \mathbb{Z}}$ of $L^{2}(\mathbb{R})$, together with a function $\varphi \in V_{0}$ is called a multiresolution analysis if it satisfies the following conditions:

(1) $V_{j} \subset V_{j+1}$ for all $j \in \mathbb{Z}$,

(2) $f \in V_{j}$ if and only if $f(2(\cdot)) \in V_{j+1}$ for all $j \in \mathbb{Z}$,

(3) $\bigcap_{j \in \mathbb{Z}} V_{j}=\{0\}$,

(4) $\overline{\bigcup_{j \in \mathbb{Z}} V_{j}}=L^{2}(\mathbb{R})$,

(5) $\{\varphi(\cdot-k): k \in \mathbb{Z}\}$ forms an orthonormal basis for $V_{0}$.

The function $\varphi$ is called a scaling function of the given MRA. An MRA gives rise to a wavelet $\psi$ which lies in the orthogonal complement of $V_{0}$ in $V_{1}$. A wavelet arising from an MRA is called an MRA wavelet. A scaling function $\varphi$ for an MRA provides a $2 \pi$-periodic function $m$, known to be the low-pass filter associated with $\varphi$ which satisfies the following:

(i) $\hat{\varphi}(2 \xi)=m(\xi) \hat{\varphi}(\xi)$,

(ii) $|m(\xi)|^{2}+|m(\xi+\pi)|^{2}=1$,

(iii) $\hat{\psi}(\xi)=e^{i \xi / 2} \overline{m\left(\frac{\xi}{2}+\pi\right)} \hat{\varphi}\left(\frac{\xi}{2}\right)$,

where the equalities above are all in the almost everywhere (a.e.) sense.

The following is a well-known characterization for a function $\varphi \in L^{2}(\mathbb{R})$ to be a scaling function.

Theorem 2.1([4]). A function $\varphi \in L^{2}(\mathbb{R})$ is a scaling function for an MRA iff

(i) $\sum_{k \in \mathbb{Z}}|\hat{\varphi}(\xi+2 k \pi)|^{2}=1$, for a.e. $\xi \in[-\pi, \pi)$,

(ii) $\lim _{j \rightarrow \infty}\left|\hat{\varphi}\left(2^{-j} \xi\right)\right|=1$, for a.e. $\xi \in \mathbb{R}$, 
(iii) there exists a $2 \pi$-periodic function $m \in L^{2}([-\pi, \pi))$ such that $\hat{\varphi}(2 \xi)=$ $m(\xi) \hat{\varphi}(\xi)$, for a.e. $\xi \in \mathbb{R}$.

From the characterization for a function $\varphi \in L^{2}(\mathbb{R})$ to be a scaling function stated as Theorem 2.1, it follows that if $\varphi$ is a scaling function, then the function $\tilde{\varphi}$ whose Fourier transform is $|\hat{\varphi}|$, is also a scaling function.

Definition 2.2([9]). A measurable function $\nu$ is called a functional wavelet multiplier if the inverse Fourier transform of $\nu \hat{\psi}$ is a wavelet, whenever $\psi$ is a wavelet.

The functional wavelet multipliers are characterized by the following theorem.

Theorem 2.2([9]). A measurable function $\nu$ is a functional wavelet multiplier iff it is unimodular and $\nu(2 t) / \nu(t)$ is a.e. equal to a $2 \pi$-periodic function.

Notation ([9]).

(1) For an MRA wavelet $\psi_{0}, S_{\psi_{0}}$ denotes the set

$$
\left\{\psi \in L^{2}(\mathbb{R}): \psi \text { is an MRA wavelet and }\left|\hat{\varphi}_{0}(\xi)\right|=|\hat{\varphi}(\xi)| \text {, a.e. }\right\},
$$

where $\varphi_{0}$ and $\varphi$ are scaling functions associated with $\psi_{0}$ and $\psi$, respectively.

(2) For a wavelet $\psi_{0}, \mathcal{M}_{\psi_{0}}$ denotes the set

$$
\left\{\psi \in L^{2}(\mathbb{R}): \hat{\psi}=\nu \hat{\psi}_{0} \text {, where } \nu \text { is a functional wavelet multiplier }\right\} \text {. }
$$

Theorem 2.3([9]). If $\psi_{0}$ is an MRA wavelet, then $S_{\psi_{0}}=\mathcal{M}_{\psi_{0}}$.

The following result related with the path-connectivity of MRA wavelets can be found in [9].

Theorem 2.4. The space $\mathcal{W}^{M}$ of all MRA wavelets is path-connected.

The proof of the above result consists of two parts. In the first part, it is proved that for a wavelet $\psi$, the class $\mathcal{M}_{\psi}$ is path-connected. In the second part, the Shannon wavelet $\psi_{0}$ is considered. For an MRA wavelet $\psi$, on account of Theorem 2.1, an appropriate element $\psi_{1} \in S_{\psi}$ is selected in such a way that it is associated with a scaling function $\varphi_{1}$ for which $\hat{\varphi}_{1} \geq 0$. If $m_{1}$ is the low-pass filter associated with the scaling function $\varphi_{1}$, then

$$
\hat{\psi}_{1}(\xi)=e^{i \xi / 2} \overline{m_{1}\left(\frac{\xi}{2}+\pi\right)} \hat{\varphi}_{1}\left(\frac{\xi}{2}\right) .
$$

The Shannon wavelet $\psi_{0}$ satisfies

$$
\hat{\psi}_{0}(\xi)=e^{i \xi / 2} \overline{m_{0}\left(\frac{\xi}{2}+\pi\right)} \hat{\varphi}_{0}\left(\frac{\xi}{2}\right),
$$


where $\hat{\varphi}_{0}(\xi)=\chi_{[-\pi, \pi)}(\xi)$ and $m_{0}$ is the low-pass filter associated with $\varphi_{0}$. Indeed, $m_{0}$ on $[-\pi, \pi)$ is given by

$$
m_{0}(\xi)=\frac{\hat{\varphi}_{0}(2 \xi)}{\hat{\varphi}_{0}(\xi)}= \begin{cases}1, & \text { if } \xi \in\left[-\frac{\pi}{2}, \frac{\pi}{2}\right), \\ 0, & \text { if } \xi \in\left[-\pi,-\frac{\pi}{2}\right) \cup\left[\frac{\pi}{2}, \pi\right) .\end{cases}
$$

For $t \in[0,1]$, a function $m_{t}$ on $[-\pi, \pi)$ is defined as follows:

$$
m_{t}(\xi)= \begin{cases}(1-t) m_{0}(\xi)+t m_{1}(\xi), & \text { if } \xi \in\left[-\frac{\pi}{2}, \frac{\pi}{2}\right) \backslash\left[-(1-t) \frac{\pi}{2},(1-t) \frac{\pi}{2}\right), \\ 1, & \text { if } \xi \in\left[-(1-t) \frac{\pi}{2},(1-t) \frac{\pi}{2}\right), \\ \sqrt{1-m_{t}^{2}(\xi+\pi)}, & \text { if } \xi \in\left[-\pi,-\frac{\pi}{2}\right), \\ \sqrt{1-m_{t}^{2}(\xi-\pi)}, & \text { if } \xi \in\left[\frac{\pi}{2}, \pi\right) .\end{cases}
$$

Extension of $m_{t}$ to a $2 \pi$-periodic function on $\mathbb{R}$ yields a low-pass filter. This family $\left\{m_{t}: t \in[0,1]\right\}$ of filters connecting $m_{0}$ to $m_{1}$ is used to define scaling functions $\left\{\varphi_{t}: t \in[0,1]\right\}$ from which the desired path-connectivity between $\psi_{0}$ and $\psi_{1}$, and hence between $\psi_{0}$ and $\psi$ has been obtained.

\section{Path-Connectedness of $\mathcal{W}_{2}$}

In [4], it has been shown that if $\psi \in L^{2}(\mathbb{R})$ is a wavelet, then $|\operatorname{supp}(\hat{\psi})| \geq$ $2 \pi$. Wavelets, whose Fourier transforms have minimal support of measure $2 \pi$ are called Minimally Supported Frequency (MSF) Wavelets by Fang and Wang [2]. Independent of Fang and Wang, Dai and Larson [1], studied the same class of wavelets and introduced the concept of wavelet sets.

A measurable set $W \subset \mathbb{R}$ is said to be a wavelet set if $|\hat{\psi}|=\chi_{W}$ for some wavelet $\psi$ in $L^{2}(\mathbb{R})$. Such a wavelet $\psi$ is called an s-elementary wavelet [1]. MSF wavelets are indeed those wavelets which are associated with wavelet sets. One of the earliest examples of wavelet sets is the Shannon or Littlewood-Paley wavelet set $[-2 \pi,-\pi) \cup[\pi, 2 \pi)$.

Ha, Kang, Lee and Seo [3], characterized wavelet sets in $\mathbb{R}$ having two intervals and proved that the wavelets arising from those two-interval wavelet sets are MRA wavelets. Such wavelets are termed to be two-interval MSF wavelets. The collection of all two-interval MSF wavelets will be denoted by $\mathcal{W}_{2}$. Wavelet sets with two intervals are precisely

$$
[2 a-4 \pi, a-2 \pi] \cup[a, 2 a],
$$

for some $0<a<2 \pi$, which we denote by $W(a)([3])$. With no loss of generality we may exclude the right end points of the intervals constituting these wavelet sets.

Observation. Consider the Shannon wavelet $\psi_{0}$, where $\left|\hat{\psi}_{0}\right|=\chi_{W(\pi)}$ and a member $\psi_{1}$ in $\mathcal{W}_{2}$, for which $\left|\hat{\psi}_{1}\right|=\chi_{W\left(\frac{\pi}{2}\right)}$. The low-pass filter $m_{0}$ for $\psi_{0}$ is given by (2.1), while that $m_{1}$ for $\psi_{1}$ is given by

$$
m_{1}(\xi)= \begin{cases}1, & \text { if } \xi \in\left[-\frac{3 \pi}{4}, \frac{\pi}{4}\right), \\ 0, & \text { if } \xi \in\left[-\pi,-\frac{3 \pi}{4}\right) \cup\left[\frac{\pi}{4}, \pi\right),\end{cases}
$$


extended $2 \pi$-periodically to $\mathbb{R}$.

These wavelets are joined by a path $\sigma:[0,1] \rightarrow \mathcal{W}^{M}$ such that the low-pass filter $m_{t}$ of $\sigma(t) \equiv \psi_{t}$ is determined by $(2.2)$.

For $t=\frac{1}{2}$,

$$
m_{1 / 2}(\xi)= \begin{cases}0, & \text { if } \xi \in\left[-\pi,-\frac{3 \pi}{4}\right), \\ \frac{\sqrt{3}}{2}, & \text { if } \xi \in\left[-\frac{3 \pi}{4},-\frac{\pi}{2}\right), \\ 1, & \text { if } \xi \in\left[-\frac{\pi}{2}, \frac{\pi}{4}\right), \\ \frac{1}{2}, & \text { if } \xi \in\left[\frac{\pi}{4}, \frac{\pi}{2}\right), \\ 0, & \text { if } \xi \in\left[\frac{\pi}{2}, \pi\right) .\end{cases}
$$

The Fourier transform of the corresponding scaling function $\varphi_{1 / 2}$ is given by

$$
\begin{aligned}
\hat{\varphi}_{1 / 2}(\xi) & =\prod_{j=1}^{\infty} m_{1 / 2}\left(2^{-j} \xi\right) \\
& = \begin{cases}0, & \text { if } \xi \in\left(-\infty,-\frac{3 \pi}{2}\right), \\
\frac{\sqrt{3}}{2}, & \text { if } \xi \in\left[-\frac{3 \pi}{2},-\pi\right), \\
1, & \text { if } \xi \in\left[-\pi, \frac{\pi}{2}\right), \\
\frac{1}{2}, & \text { if } \xi \in\left[\frac{\pi}{2}, \pi\right), \\
0, & \text { if } \xi \in[\pi, \infty),\end{cases}
\end{aligned}
$$

and the Fourier transform of the wavelet $\psi_{1 / 2}$ is given by

$$
\begin{aligned}
\hat{\psi}_{1 / 2}(\xi)= & e^{\frac{i \xi}{2}} \overline{m_{1 / 2}\left(\frac{\xi}{2}+\pi\right)} \hat{\varphi}_{1 / 2}\left(\frac{\xi}{2}\right) \\
= & \begin{cases}0, & \text { if } \xi \in(-\infty,-3 \pi) \cup[2 \pi, \infty), \\
\frac{\sqrt{3}}{2} e^{\frac{i \xi}{2}}, & \text { if } \xi \in[-3 \pi,-2 \pi), \\
e^{\frac{i \xi}{2}}, & \text { if } \xi \in\left[-2 \pi,-\frac{3 \pi}{2}\right), \\
\frac{1}{2} e^{\frac{i \xi}{2}}, & \text { if } \xi \in\left[-\frac{3 \pi}{2},-\pi\right), \\
0, & \text { if } \xi \in\left[-\pi, \frac{\pi}{2}\right), \\
\frac{\sqrt{3}}{2} e^{\frac{i \xi}{2}}, & \text { if } \xi \in\left[\frac{\pi}{2}, \pi\right), \\
\frac{1}{2} e^{\frac{i \xi}{2}}, & \text { if } \xi \in[\pi, 2 \pi) .\end{cases}
\end{aligned}
$$

Clearly, the wavelet $\psi_{1 / 2}$ does not lie in $\mathcal{W}_{2}$. Indeed, computation can be made to check that no point of the path $\sigma$ other than $\sigma(0)$ and $\sigma(1)$ lies in $\mathcal{W}_{2}$.

Now we prove that $\mathcal{W}_{2}$ is path-connected. The technique of the proof involves adjustment of the intermediate filters which join the low-pass filter $m_{0}$ of the Shannon wavelet to the low-pass filter $m_{a}^{1}$ of a two-interval MSF wavelet $\psi_{a}^{1}$ with $\operatorname{supp}\left(\hat{\psi}_{a}^{1}\right)=[2 a-4 \pi, a-2 \pi) \cup[a, 2 a)$, keeping the path joining $\psi_{0}$ and $\psi_{a}^{1}$ in $\mathcal{W}_{2}$. Here it may be noticed that if $\psi_{0}$ is a two-interval MSF wavelet and $\left|\hat{\psi}_{0}\right|=\chi_{W(a)}$, where $a \in(0,2 \pi)$, then for each member $\psi$ of $\mathcal{M}_{\psi_{0}},|\hat{\psi}|=\chi_{W(a)}$.

Theorem 3.1. The space $\mathcal{W}_{2}$ is path-connected. 
Proof. Let $\psi_{0}$ be the Shannon wavelet. Then the low-pass filter $m_{0}$ for the Shannon wavelet is given by $(2.1)$.

Suppose $\psi_{a}$ is a two-interval MSF wavelet given by

$$
\left|\hat{\psi}_{a}(\xi)\right|=\chi_{W(a)}(\xi),
$$

where $0<a<2 \pi$. Then the modulus of the Fourier transform of the scaling function $\varphi_{a}$ comes out to be

$$
\left|\hat{\varphi}_{a}(\xi)\right|=\chi_{[a-2 \pi, a)}(\xi) .
$$

Choose $\psi_{a}^{1} \in S_{\psi_{a}}=\mathcal{M}_{\psi_{a}}$ such that it is associated with the scaling function $\varphi_{a}^{1}$, whose Fourier transform is given by

$$
\hat{\varphi}_{a}^{1}=\left|\hat{\varphi}_{a}\right|=\chi_{[a-2 \pi, a)} .
$$

Clearly $\hat{\varphi}_{a}^{1} \geq 0$. The low-pass filter $m_{a}^{1}$ associated with $\varphi_{a}^{1}$ on $[a-2 \pi, a)$ is given by

$$
m_{a}^{1}(\xi)=\frac{\hat{\varphi}_{a}^{1}(2 \xi)}{\hat{\varphi}_{a}^{1}(\xi)}= \begin{cases}1, & \text { if } \xi \in\left[\frac{a-2 \pi}{2}, \frac{a}{2}\right), \\ 0, & \text { if } \xi \in\left[a-2 \pi, \frac{a-2 \pi}{2}\right) \cup\left[\frac{a}{2}, a\right),\end{cases}
$$

which is then extended $2 \pi$-periodically to $\mathbb{R}$.

Also, we have

$$
\left|\hat{\psi}_{a}^{1}(\xi)\right|=\chi_{W(a)}(\xi) .
$$

Now, for each $s \in[0,1]$, we define the function $m_{a}^{s}$ on $[-\pi+(a-\pi) s, \pi+(a-\pi) s)$ as follows:

$m_{a}^{s}(\xi)= \begin{cases}1, & \text { if } \xi \in\left[-\frac{\pi}{2}+\frac{(a-\pi) s}{2}, \frac{\pi}{2}+\frac{(a-\pi) s}{2}\right), \\ 0, & \text { if } \xi \in\left[-\pi+(a-\pi) s,-\frac{\pi}{2}+\frac{(a-\pi) s}{2}\right) \cup\left[\frac{\pi}{2}+\frac{(a-\pi) s}{2}, \pi+(a-\pi) s\right),\end{cases}$

and extend it $2 \pi$-periodically to the whole of $\mathbb{R}$. For $\xi \in \mathbb{R}$, we set

$$
\hat{\varphi}_{a}^{s}(\xi)=\prod_{j=1}^{\infty} m_{a}^{s}\left(2^{-j} \xi\right) .
$$

Since $0 \leq m_{a}^{s}(\xi) \leq 1$, this product is well-defined. For

$$
\xi \in\left[2^{j}(\pi+(a-\pi) s), 2^{j+1}(\pi+(a-\pi) s)\right), \text { with } j \geq 0,
$$

we have

$$
t \equiv 2^{-(j+1)} \xi \in\left[\frac{\pi}{2}+\frac{(a-\pi) s}{2}, \pi+(a-\pi) s\right),
$$

and hence $m_{a}^{s}(t)=0$. Thus

$$
\begin{aligned}
\hat{\varphi}_{a}^{s}(\xi) & =\hat{\varphi}_{a}^{s}\left(2^{j+1} t\right)=m_{a}^{s}\left(2^{j} t\right) \hat{\varphi}_{a}^{s}\left(2^{j} t\right) \\
& =m_{a}^{s}\left(2^{j} t\right) m_{a}^{s}\left(2^{j-1} t\right) \cdots m_{a}^{s}(t) \prod_{l=1}^{\infty} m_{a}^{s}\left(2^{-l} t\right)=0 .
\end{aligned}
$$


Therefore, $\hat{\varphi}_{a}^{s}(\xi)=0$ for all $\xi \in[\pi+(a-\pi) s, \infty)$. Similarly, we can show that if $\xi \in$ $(-\infty,-\pi+(a-\pi) s)$, then $\hat{\varphi}_{a}^{s}(\xi)=0$. Thus $\operatorname{supp}\left(\hat{\varphi}_{a}^{s}\right) \subseteq[-\pi+(a-\pi) s, \pi+(a-\pi) s]$. In fact, $\hat{\varphi}_{a}^{s}=\chi_{[-\pi+(a-\pi) s, \pi+(a-\pi) s)}$. Now, we show that $\varphi_{a}^{s}$ is a scaling function for an MRA. For this we use Theorem 2.1. By the definition of $\hat{\varphi}_{a}^{s}$, it is clear that $\hat{\varphi}_{a}^{s}(2 \xi)=$ $m_{a}^{s}(\xi) \hat{\varphi}_{a}^{s}(\xi)$ for all $\xi \in \mathbb{R}$. Further, $\hat{\varphi}_{a}^{s}(\xi)=1$, when $\xi \in[-\pi+(a-\pi) s, \pi+(a-\pi) s)$ and $0 \leq s \leq 1$. Therefore, $\varphi_{a}^{s}$ satisfies conditions (ii) and (iii). For condition (i), let $S=[-\pi+(a-\pi) s, \pi+(a-\pi) s)$. We easily see that the set $\{S-2 k \pi: k \in \mathbb{Z}\}$ partitions $\mathbb{R}$, and therefore for a.e. $\xi \in \mathbb{R}$, there exists exactly one $k \in \mathbb{Z}$ such that $\xi+2 k \pi \in S$. Thus condition (i) follows.

Using the relation

$$
\hat{\psi}_{a}^{s}(\xi)=e^{i \xi / 2} \overline{m_{a}^{s}\left(\frac{\xi}{2}+\pi\right)} \hat{\varphi}_{a}^{s}\left(\frac{\xi}{2}\right)
$$

we have

$$
\left|\hat{\psi}_{a}^{s}(\xi)\right|=\chi_{[-2 \pi+2(a-\pi) s,-\pi+(a-\pi) s) \cup[\pi+(a-\pi) s, 2 \pi+2(a-\pi) s)}(\xi),
$$

with $0<\pi+(a-\pi) s<2 \pi$.

Thus $\psi_{a}^{s}$ is a two-interval MSF wavelet. The proof of the continuity of the map $s \mapsto \psi_{a}^{s}$ is drawn on almost the similar lines as that of $s \mapsto \psi_{s}$ described in [9], by observing the following:

(i) For a fixed $s \in[0,1]$ the set of points $\xi \in \mathbb{R}$, for which the map $t \mapsto m_{a}^{t}\left(2^{-j} \xi\right)$, $j \geq 1$ is not continuous at $s$, is countable.

(ii) For $0<a \leq \pi, \hat{\varphi}_{a}^{t}(\xi)=\hat{\varphi}_{a}^{1}(\xi)$ on $[-a, a)$, for all $t \in[0,1]$.

(iii) For $\pi \leq a<2 \pi, \hat{\varphi}_{a}^{t}(\xi)=\hat{\varphi}_{a}^{1}(\xi)$ on $[-(2 \pi-a),(2 \pi-a))$, for all $t \in[0,1]$.

Thus, we have a path $\gamma_{a}:[0,1] \rightarrow \mathcal{W}_{2}$ defined by $\gamma_{a}(s)=\psi_{a}^{s}$, joining $\psi_{0} \equiv \psi_{a}^{0}$ to $\psi_{a}^{1}$, and hence with $\psi_{a}$ as each $\mathcal{M}_{\psi_{a}}$ is path-connected.

Acknowledgements The author thanks to Professor K. K. Azad for his help and encouragement.

\section{References}

[1] X. Dai and D. R. Larson, Wandering vectors for unitary systems and orthogonal wavelets, Mem. Amer. Math. Soc., 134(1998), no. 640, MR 98m: 47067.

[2] X. Fang and X. Wang, Construction of minimally-supported-frequency wavelets, J. Fourier Anal. Appl., 2(1996), 315-327.

[3] Y. Ha, H. Kang, J. Lee and J. K. Seo, Unimodular wavelets for $L^{2}$ and the Hardy space $H^{2}$, Michigan Math. J., 41(1994), 345-361. 
[4] E. Hernández and G. Weiss, A First Course on Wavelets, CRC Press, 1996.

[5] Z. Li, X. Dai, Y. Diao and W. Huang, The Path-connectivity of MRA wavelets in $L^{2}\left(\mathbb{R}^{d}\right)$, Illinois J. Math., to appear.

[6] Z. Li, X. Dai, Y. Diao and J. Xin, Multipliers, phases and connectivity of MRA wavelets in $L^{2}\left(\mathbb{R}^{2}\right)$, J. Fourier Anal. Appl., 16(2010), 155-176.

[7] S. G. Mallat, Multiresolution approximations and wavelet orthonormal bases of $L^{2}(\mathbb{R})$, Trans. Amer. Math. Soc., 315(1989), 69-87.

[8] D. Speegle, The s-elementary wavelets are path-connected, Proc. Amer. Math. Soc., 127(1999), 223-233.

[9] The Wutam Consortium, Basic properties of wavelets, J. Fourier Anal. Appl., 4(1998), 575-594. 